\title{
The index and co-index of the twisted tangent bundle over projective spaces
}

\author{
RYUICHI TANAKA*
}

\begin{abstract}
We determine the index and co-index of the twisted tangent bundle of projective spaces. We also discuss the stabilty of them, and determine the set of integers that can be realized as the stable co-index of a vector bundle over the projective space.
\end{abstract}

\section{Introduction.}

Let $\alpha$ be a finite-dimensional real vector bundle over a CW complex $B$, and let $S(\alpha)$ be its sphere bundle with respect to some metric on $\alpha$. We regard $S(\alpha)$ as a $\mathbb{Z} / 2$-space by the antipodal map on each fibre. Then, the index of $\alpha$, denoted ind $\alpha$, is defined to be the largest integer $k$ for which there exists a $\mathbb{Z} / 2$-map from $S^{k-1}$ to $S(\alpha)$. Here, $S^{k-1}$ also is equipped with a $\mathbb{Z} / 2$-action by the antipodal map. As a dual notion to the index, the co-index of $\alpha$, denoted co-ind $\alpha$, is defined to be the smallest integer $k$ for which there exists a $\mathbb{Z} / 2$-map from $S(\alpha)$ to $S^{k-1}$. If there exist no such maps, we define co-ind $\alpha$ as $\infty$. When $B$ is a finite complex, co-ind $\alpha$ is finite for any $\alpha$. By the Borsuk-Ulam theorem, ind $\alpha$ is not greater than co-ind $\alpha$.

In this paper, we calculate the index and co-index of the twisted tangent bundle of the real projective space $\mathbb{R} P^{n}$. Let $\tau$ denote the tangent bundle of $\mathbb{R} P^{n}$, and let $\xi$ denote the canonical line bundle over $\mathbb{R} P^{n}$. We call $\tau \otimes \xi$ the twisted tangent bundle of $\mathbb{R} P^{n}$. For the tangent bundle $\tau$, we already know that ind $\tau=n$, since the index of the tangent bundle of any closed manifold is equal to its dimension [CF1, Theorem 6.11]. As for the co-index of $\tau$, it is known that co-ind $\tau$ is equal to $l(n)(n \neq 1,3,7)$ or $l(n)-1(n=1,3,7)$, where $l(n)$ denotes the smallest integer $m$ such that $\mathbb{R} P^{n}$ can be immersed in $\mathbb{R}^{m}$ [AGJ, T1].

We shall prove the following results in which the $k$-dimensional trivial bundle is denoted simply by $k$.

Theorem 1.1. For $\tau \otimes \xi$, the twisted tangent bundle of $\mathbb{R} P^{n}$, we have co-ind $(\tau \otimes \xi+k)=n+1+k$ for any $k \geq 0$.

Received August 10, 2004; revised August 26, 2004.

2000 Mathematics Subject Classification. Primary 55P91 ; Secondary 55R25.

* Department of Liberal Arts, Faculty of Science and Technology, Tokyo University of Science, Noda, Chiba, 278-8510, Japan.

e-mail: 
Theorem 1.2. For $\tau \otimes \xi$, the twisted tangent bundle of $\mathbb{R} P^{n}$, we have the following.

(1) If $n$ is odd, then $\operatorname{ind}(\tau \otimes \xi)=n+1$ and $\operatorname{ind}(\tau \otimes \xi+k)=n+k$ for $k \geq 1$.

(2) If $n$ is even, then $\operatorname{ind}(\tau \otimes \xi+k)=n+k$ for any $k \geq 0$.

For a vector bundle $\alpha$, in general, we call $\alpha$ to be $C I$-stable if the equality co-ind $(\alpha+k)=$ co-ind $\alpha+k$ holds for any positive integer $k$. Likewise we call $\alpha$ to be $I$-stable if ind $(\alpha+k)=$ ind $\alpha+k$ for any positive integer $k$. We have the following corollaries.

Corollary 1.3. $\tau \otimes \xi$ is CI-stable.

\section{Corollary 1.4 .}

(1) If $n$ is odd, then $\tau \otimes \xi$ is not I-stable but $\tau \otimes \xi+1$ turns to be I-stable.

(2) If $n$ is even, then $\tau \otimes \xi$ is I-stable.

As for $\tau$, the tangent bundle of $\mathbb{R} P^{n}$, it is not known whether $\tau$ is CI-stable, though the equality co-ind $(\tau+1)=$ co-ind $\tau+1$ holds as shown in [T1, Theorem 3.7]. On the other hand, $\tau$ is I-stable, since the tangent bundle of any closed manifold is known to be I-stable [T2, Theorem 4.6]. Also, it is to be noted that $\xi$ is not I-stable, but CI-stable [T1, Theorem 4.1].

For $\alpha$, a vector bundle over a finite complex $B$, we can define the stable co-index of $\alpha$, denoted co-ind ${ }^{s}(\alpha)$, as

$$
\operatorname{co-ind}^{s}(\alpha)=\underset{k}{\lim }\{\operatorname{co-ind}(\alpha+k)-\operatorname{dim}(\alpha+k)\} \text {. }
$$

This limit always exists since $0 \leq \operatorname{co-ind}(\alpha+1)-\operatorname{dim}(\alpha+1) \leq \operatorname{co-ind} \alpha-\operatorname{dim} \alpha$ (see $\left[\mathrm{T} 1\right.$, Lemma 3.1]). Likewise we define the stable index $\operatorname{ind}^{s}(\alpha)$. For example, if $\alpha$ is stably trivial, then, $\operatorname{co-ind}^{s}(\alpha)=\operatorname{ind}^{s}(\alpha)=0$. Also, we know that co-ind $^{s}(\xi)=n$ and ind $^{s}(\xi)=0$ from [T1, Theorem 4.1]. The above theorems imply co-ind ${ }^{s}(\tau \otimes \xi)=1$ and ind $^{s}(\tau \otimes \xi)=0$. For the $m$-fold sum of the twisted tangent bundle $\tau \otimes \xi$, we shall prove the following theorem.

Theorem 1.5. If $m \leq n$, then co-ind ${ }^{s}(m \cdot \tau \otimes \xi)=m$.

If $\alpha$ is stably equivalent to $\alpha^{\prime}$, it is clear that co-ind ${ }^{s}(\alpha)=\operatorname{co-ind}^{s}\left(\alpha^{\prime}\right)$. So, co-ind $^{s}$ can be considered as a function from $\widetilde{K O}(B)$ to $\mathbb{Z}^{+}$, the set of nonnegative integers. Since co-ind $\alpha \leq \operatorname{dim} \alpha+\operatorname{dim} B$ ([T1, Lemma 3.1]), we have $\operatorname{co-ind}^{s}(\alpha) \leq \operatorname{dim} B$ in general. Thus, $\operatorname{dim} B$ is an upper bound of the image of the function co-ind ${ }^{s}$. From Theorem 1.5, we obtain the following corollary.

Corollary 1.6. The image of the function co-ind ${ }^{s}: \widetilde{K O}\left(\mathbb{R} P^{n}\right) \longrightarrow \mathbb{Z}^{+}$is equal to the set $\{0,1,2, \cdots, n\}$.

Thus, over $\mathbb{R} P^{n}$, any non-negative integer not greater than $\operatorname{dim} \mathbb{R} P^{n}$ can be realized as the stable co-index of a vector bundle.

\section{Proof of the theorems.}

Let $B$ be a finite complex and $\alpha$ be a real vector bundle over $B$. We define the $\nu$-dimension of $\alpha$ to be the smallest integer $k$ for which there is a $k$-dimensional 
vector bundle $\beta$ with $\alpha+\beta$ trivial, and we denote it by $\nu$-dim $\alpha$. The $\nu$-dimension of $\alpha$ is closely related to the geometric dimension of $-\alpha$, but they slightly differ in general (see examples in [T2]). The inequalities g-dim( $(-\alpha) \leq \nu$-dim $\alpha \leq \operatorname{dim} B$ hold generally. We easily see that co-ind $\alpha \leq \operatorname{dim} \alpha+\nu$-dim $\alpha$.

For a virtual vector bundle $\alpha$, we denote by $\omega$-dim $\alpha$ the largest integer $k$ for which the $k$ th Stiefel-Whitney class $w_{k}(\alpha)$ is not zero. Then it is known that co-ind $\alpha \geq \operatorname{dim} \alpha+\omega-\operatorname{dim}(-\alpha)$ and the following theorem holds.

Theorem 2.1([T2, Theorem 2.5]). Let $\alpha$ be a real vector bundle over a finite complex $B$, and suppose that the equality $\omega-\operatorname{dim}(-\alpha)=\nu$-dim $\alpha$ holds. Then,

(1) co-ind $\alpha=\operatorname{dim} \alpha+\nu$-dim $\alpha$.

(2) $\alpha$ is CI-stable.

Now, we prove Theorem 1.1 and Theorem 1.5.

Proof of Theorem 1.1 and 1.5.

Since $\tau+1 \cong(n+1) \xi$, we have $\tau \otimes \xi+\xi \cong n+1$. Hence, $\nu$-dim $(\tau \otimes \xi) \leq 1$. We also have $W(-\tau \otimes \xi)=W(\xi)=1+t$ where $W$ is the total Stiefel-Whitney class and $t$ is the generator of $H^{*}\left(\mathbb{R} P^{n} ; \mathbb{Z} / 2\right)$. Hence, $\omega-\operatorname{dim}(-\tau \otimes \xi)=1$. Since $\omega-\operatorname{dim}(-\alpha) \leq \nu$-dim $\alpha$ in general, we have $\omega-\operatorname{dim}(-\tau \otimes \xi)=\nu$-dim $(\tau \otimes \xi)=1$. Therefore, from Theorem 2.1, we find that co-ind $(\tau \otimes \xi)=n+1$, and also, $\tau \otimes \xi$ is CI-stable. Thus Theorem 1.1 follows. Our argument goes as well for the $m$-fold sum of the twisted tangent bundle $\tau \otimes \xi$. In fact, since $m \cdot \tau \otimes \xi+m \xi \cong m(n+1)$, we have $\nu$-dim $(m \cdot \tau \otimes \xi) \leq m$. On the other hand, since $W(-m \cdot \tau \otimes \xi)=$ $W(m \xi)=(1+t)^{m}$, we have $\omega-\operatorname{dim}(-m \cdot \tau \otimes \xi)=m$ if $m \leq n$. Thus, if $m \leq n$ we see that $\omega-\operatorname{dim}(-m \cdot \tau \otimes \xi)=\nu-\operatorname{dim}(m \cdot \tau \otimes \xi)=m$, and, from Theorem 2.1, it follows that $m \cdot \tau \otimes \xi$ is CI-stable and co-ind ${ }^{s}(m \cdot \tau \otimes \xi)=m$.

Next, we turn to the index. To prove Theorem 1.2, we use the following result in [T1].

Proposition 2.2([T1, Corollary 2.5]). Let $\alpha$ be a real vector bundle over $\mathbb{R} P^{n}$.

(1) If $\operatorname{dim} \alpha>n$, then ind $\alpha=\operatorname{dim} \alpha$.

(2) If $\operatorname{dim} \alpha \leq n$ and $\alpha$ contains $\xi$ as a subbundle, then ind $\alpha=n+1$.

Proof of Theorem 1.2.

In view of Proposition $2.2(1)$, we have only to prove that $\operatorname{ind}(\tau \otimes \xi)$ is equal to $n+1$ if $n$ is odd, and $n$ if $n$ is even. Since ind $\alpha \leq$ co-ind $\alpha$ in general, we have $\operatorname{ind}(\tau \otimes \xi) \leq n+1$ from Theorem 1.1. Also, since ind $\alpha \geq \operatorname{dim} \alpha$ in general, we clearly have $\operatorname{ind}(\tau \otimes \xi) \geq n$. Now, suppose that there is a $\mathbb{Z} / 2$ map $f: S^{n} \longrightarrow S(\tau \otimes \xi)$. Let $\tilde{f}: \mathbb{R} P^{n} \longrightarrow P(\tau \otimes \xi)$ be the map induced from $f$. Here, $P(\tau \otimes \xi)$ is the projective bundle associated to $\tau \otimes \xi$. Let $p$ : $P(\tau \otimes \xi) \longrightarrow \mathbb{R} P^{n}$ be the bundle projection. Let $e$ denote the $\mathbb{Z} / 2$-Euler class of the bundle $S(\tau \otimes \xi) \longrightarrow P(\tau \otimes \xi)$. We consider the homomorphism $\tilde{f}^{*}$ : $H^{*}(P(\tau \otimes \xi) ; \mathbb{Z} / 2) \longrightarrow H^{*}\left(\mathbb{R} P^{n} ; \mathbb{Z} / 2\right)$. From the naturality of the Euler class, we have $\tilde{f}^{*}(e)=t$, and so, $\tilde{f}^{*}\left(e^{n}\right)=t^{n}$, which is not zero. On the other hand, in $H^{*}(P(\tau \otimes \xi) ; \mathbb{Z} / 2)$, we have the relation

$$
e^{n}=w_{n}+w_{n-1} e+w_{n-2} e^{2}+\cdots+w_{1} e^{n-1},
$$


where $w_{i}$ 's are the Stiefel-Whitney classes of $\tau \otimes \xi$ and $H^{*}(P(\tau \otimes \xi) ; \mathbb{Z} / 2)$ is considered as a $H^{*}\left(\mathbb{R} P^{n} ; \mathbb{Z} / 2\right)$-module in an obvious way. Since $\tau \otimes \xi+\xi \cong n+1$, we have $W(\tau \otimes \xi)=W(\xi)^{-1}=(1+t)^{-1}$. Hence, we have the relation

$$
e^{n}=t^{n}+t^{n-1} e+t^{n-2} e^{2}+\cdots+t e^{n-1} .
$$

Applying $\tilde{f}^{*}$, we have

$$
t^{n}=(p \circ \tilde{f})^{*}\left(t^{n}\right)+(p \circ \tilde{f})^{*}\left(t^{n-1}\right) t+(p \circ \tilde{f})^{*}\left(t^{n-2}\right) t^{2}+\cdots+(p \circ \tilde{f})^{*}(t) t^{n-1} .
$$

But the homomorphism $(p \circ \tilde{f})^{*}: H^{*}\left(\mathbb{R} P^{n} ; \mathbb{Z} / 2\right) \longrightarrow H^{*}\left(\mathbb{R} P^{n} ; \mathbb{Z} / 2\right)$ is either identity or zero. The latter case is impossible since $t^{n}$ is not zero. Therefore, we have $t^{n}=n t^{n}$. If $n$ is even, this contradicts $t^{n} \neq 0$. Thus we have proved that $\operatorname{ind}(\tau \otimes \xi)$ is equal to $n$ if $n$ is even.

Now, let $n$ be odd. Then $\tau$ has a non-zero cross-section since it is orientable and its Euler class is zero. In other words, $\tau$ has a one-dimensional trivial bundle as a subbundle. Hence $\tau \otimes \xi$ has $\xi$ as a subbundle. From Proposition $2.2(2)$, it follows that ind $(\tau \otimes \xi)=n+1$. This completes the proof.

\section{REFERENCES}

[AGJ] J.Adem, S.Gitler and I.M.James, On axial maps of a certain type, Bol. Soc. Mat. Mex. 17 (1972), 59-62.

[CF1] P.E.Conner and E.E.Floyd, Fixed point free involutions and equivariant maps, Bull. Amer. Math. Soc. 66 (1960), 416-441.

[CF2] _ Fixed point free involutions and equivariant maps II, Trans. Amer. Math. Soc. 105 (1962), 222-228.

[S] S.Stolz, The level of real projective spaces, Comment. Math. Helv. 64 (1989), 661-674.

[T1] R.Tanaka, On the index and co-index of sphere bundles, Kyushu J. Math. 57 (2003), 371-382.

[T2] $321-331$. 